\title{
Computation of Rectifier Transformers employed in Railway Networks
}

\author{
Gholamhossein Shirkoohi, Member, IEEE and Alex Jenkins \\ School of Engineering, London South Bank Univesity, \\ London SE1 OAA, UK, email: maziar.shirkoohi@Isbu.ac.uk
}

\begin{abstract}
Many rail transport providers operate and maintain a medium voltage distribution network providing power to trains, signalling equipment and stations. Transformers form an important part of the distribution network stepping voltage up and down. Rectifier transformers are used to provide DC for traction, usually stepping down from medium voltage levels. Mineral oil is essential for optimised performance of large transformers, providing the much needed cooling at high power operation and elevated core and winding temperatures. A representative rectifier transformer has been modelled for a realistic analysis of the $3 \mathrm{D}$ model constructed using exact manufacturer's data for the geometry and coil arrangements. The results are seen to be in good agreement with the actual tests carried out during the commissioning of the transformer.
\end{abstract}

Index Terms - Rectifier transformer, rail transport, Transformer characteristics, Oil filled transformer.

\section{INTRODUCTION}

A large proportion of distribution transformers around the world are filled with mineral oil. Mineral oil acts as an insulator and coolant aiding the transformer to function reliably and efficiently. Transformer mineral oil is made by refining a fraction of the hydro-carbons during the distillation of a petroleum crude stock. The boiling range of the collected fraction and the type and degree of the refining process are selected so that the resulting oil has the desired characteristics [1-2].

Mineral oil is extremely popular and widely used because it is relatively cheap, readily available, proven product reliability and has good physical, chemical and electrical properties. However, it is non-biodegradable, water hazardous, flammable, corrosive sulphur and compared to newer alternatives have low fire point and moisture tolerance. Where it is initially cheaper installing oil spillage preventatives drives the cost up significantly.

Railway operators purchase and arrange installation of rectifier transformers. Each substation location is unique and provides project teams with different delivery issues. The rail companies do not usually generate electricity and are therefore reliant on grid points to provide a reliable supply. The supply voltage from the grid is dependent on the location however commonly a 3 phase, $33 \mathrm{kV}, 50 \mathrm{~Hz}$ supply (in the UK) is provided. To provide power to trains an AC distribution network is maintained and operated by electrical control rooms and maintenance staff. Substations are located 3-4 miles apart with an open point between two different Grid supplies. This prevents the two supplies from being connected in parallel and provides an element of diversity if a fault occurs as the open point can be moved to maintain the network. Transmission cables are laid in the ground and connect substations together. They are uniquely identified by high voltage (HV) feeder cable numbers. Feeder cables enter the substation and terminate onto an AC circuit breaker which has the same identification number. The circuit is maintained through circuit breakers by busbars and distributed to the next substation via other feeder cables.

AC circuit breakers can be operated remotely by Electrical Control Rooms or locally by competent staff. They have intelligent fault protection systems which operates the circuit breaker under fault conditions. They are imperative for the following reasons; isolate faulty equipment, and to divide large electrical networks into smaller sections reconfigure the network in order to restore power supplies and control other electrical assets. The fundamental ratings for switchgear are outlined by specific regulations. In this case, the rated normal current of circuit breakers and all busbars is $400 \mathrm{~A}, 630 \mathrm{~A}$, $800 \mathrm{~A}$ or $1250 \mathrm{~A}$. The short circuit current rating of the equipment should be a minimum of the following, or as specified, in this case; $13.0 \mathrm{kA}$ for 3 seconds for $11 \mathrm{kV}$ systems, $17.5 \mathrm{kA}$ for 3 seconds for $22 \mathrm{kV}$ systems and $17.5 \mathrm{kA}$ for 3 seconds for $33 \mathrm{kV}$ systems. This means the equipment must be able to withstand $17.5 \mathrm{kA}$ for a minimum of 3 seconds without mechanical or physical damage occurring for $33 \mathrm{kV}$ systems and rated for a normal current of 1250A. Fig. 1 shows a typical transformer in this category, positioned in its final location with arrangements made for bonding trench, in anticipation of any oil leak, before installation of the mesh flooring and the rain shelter housing.

A substation with two feeder cables has three AC circuit breakers. Each feeder cable will have a circuit breaker as does the transformer. The rectifier circuit breaker is connected to an oil filled step down transformer (the type under investigation here) which is located outside. The transformer is connected to a rectifier via anode cables. The rectifier converts $\mathrm{AC}$ voltage to $\mathrm{DC}$ voltage. The main DC circuit breaker forms part of the 
DC switchgear inside the substation. The number of DC track circuit breakers depends on how many railway lines the substation is supplying. If there are two railway lines there will be four DC track circuit breakers. DC circuit breakers are required for similar reasons to $\mathrm{AC}$ circuit breakers. Positive track feeder cables are terminated onto each DC track circuit breaker and run outside onto the track and terminate on to a specific length of conductor rail. The conductor rail is mounted on insulators to prevent any leakage current to earth. Trains have shoe gear mounted at their base which slides along the conductor rail providing power to the train. Section gaps are strategically located by substations to divide the conductor rail into sub-sections. Substation electrical equipment and inter wiring is shown in Figure 2.

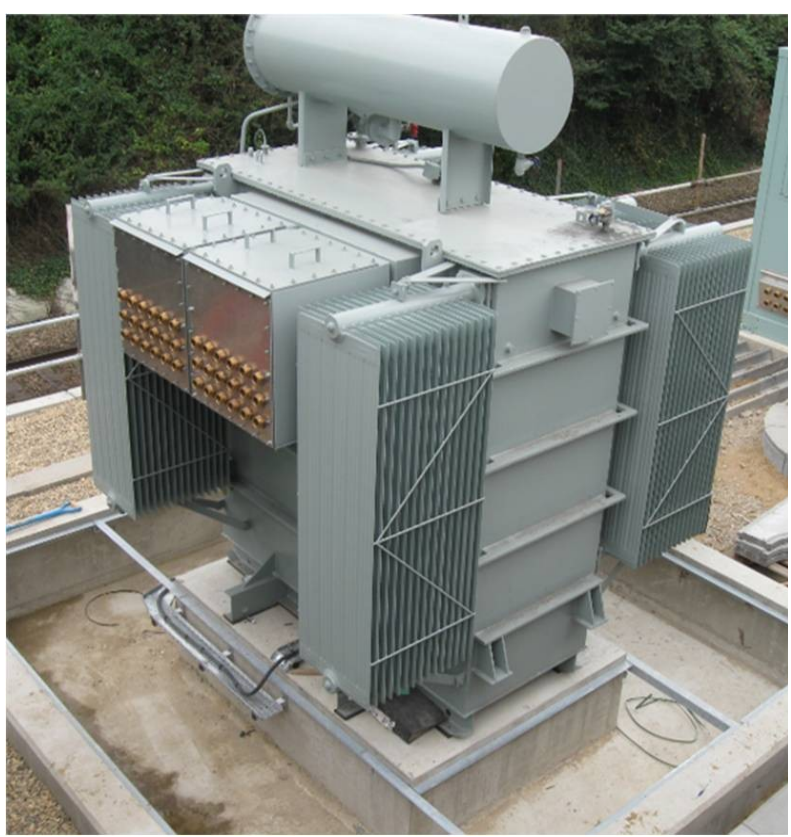

Fig. 1. Transformer in its Final Location with arrangements made for bonding, prior to the installation of the rain shelter.

\section{RECTIFIER TRANSFORMER AND RECTIFIER UNIT}

The rectifier transformers are located outside and the rectifier circuits inside. The two pieces of equipment are connected by cable terminated from the low voltage (LV) secondary of the transformer and $\mathrm{AC}$ side of the rectifier. Transformers are used to step voltage down, and rectifiers to convert it from $\mathrm{AC}$ into $\mathrm{DC}$ volts.

All rectifier transformers in the Region have vector groups specifying the phase displacement between the primary and secondary windings. HV windings are identified by capital letters $(\mathrm{D}, \mathrm{Y}, \mathrm{Z})$ and $\mathrm{LV}$ windings by lower case letters $(\mathrm{d}, \mathrm{y}, \mathrm{z})$.

The rectifier transformers are usually configured Dy11d0. They have one primary HV Delta winding and two secondary LV windings, star and delta, thus it is a step down transformer.

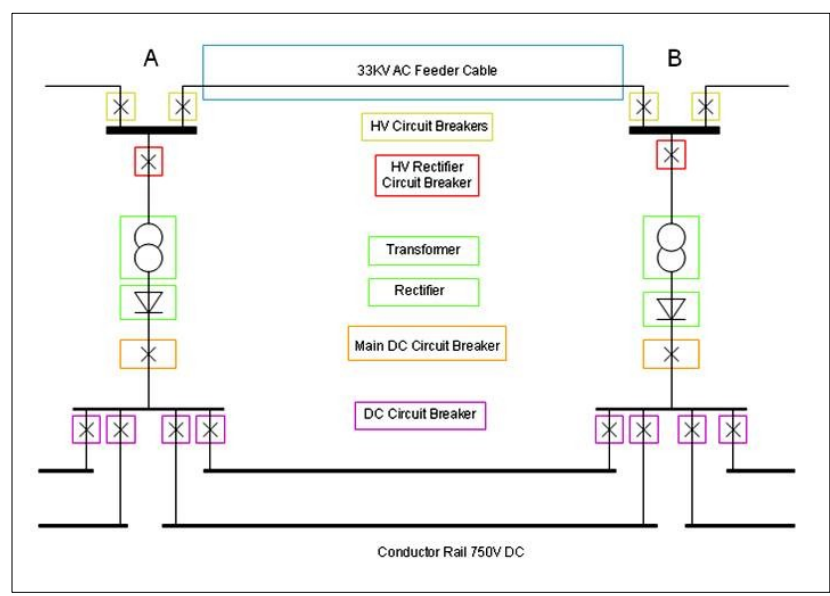

Fig. 2. Electrical Components within a Substation Compound

The numbers relate to the phase displacement between the primary and secondary windings. It is possible to use numbers $1-12$ set in the same position as a clock face to represent $360^{\circ}$. Number 12 is set at $0^{\circ}$ and there is $30^{\circ}$ between each of the numbers. The HV winding is always used as the reference point at $0^{\circ}$. The rectifier transformer is manufactured so that the star LV secondary winding leads the $\mathrm{HV}$ winding by $30^{\circ}$ and hence, there is no phase displacement between the delta LV secondary winding and the HV winding. The transformer has 3 coils inside its tank, one for each phase. Coils are wound in a unique way so the HV and two LV's are on the same limb. Construction starts with the LV windings being wound around the centre.

Delta and star LV windings are insulated from one another. HV windings are wrapped around the LV windings with an added layer of insulation between them. LV windings are usually foil wound and HV windings disc wound. Figure 3 shows the low voltage (LV) windings of the transformer being wound, which are usually made from wound copper foil. The three completed outer HV windings can also be seen in Figure 4 , where a common laminated iron core passes through the center of each winding.

Rectifier transformers have the capability to change the primary voltage in order to maintain the correct secondary voltage. This is done via a tap changer which is wound on the HV winding. They have a 3 phase input and 6 phase output. Two LV windings both output 293.7 Volts. To obtain equal

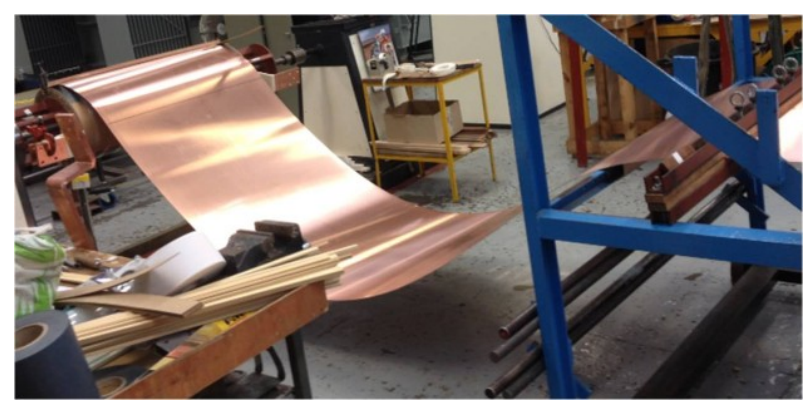

Fig. 3. The low voltage (LV) windings are usually made from wound copper foil. 


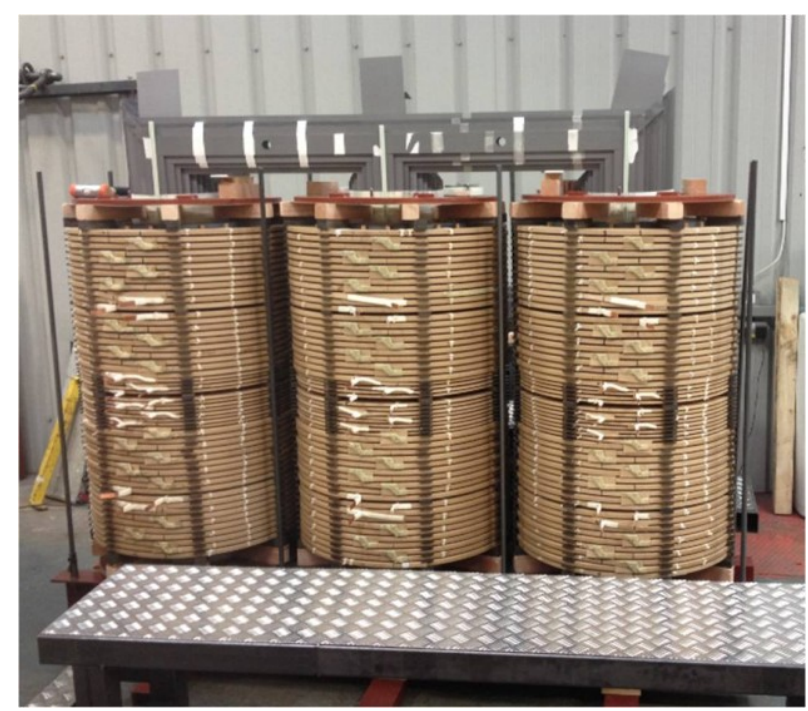

Fig. 4. Three completed (three phase) windings, high voltage (HV) discs on the outside of the core.

secondary voltages, the number of turns of the secondary windings must be in the ratio of $1: \sqrt{3}$. Since $\sqrt{3}$ is an irrational number the turn ratio of the two secondary windings can only be approximated. Common ratios are 4:7 or 7:12.

These transformers are categorised as Class $\mathrm{G}$ and must conform to BS 4417: 1969 [3]. In the standard states Class G transformers duty cycle should usually be specified by the purchaser. Modern trains draw current when they accelerate. The amount of current is dependent on the rolling stock and number of train carriages. The load seen by the transformer is determined by the number of trains accelerating at the same time in the same electrical section. Peak load occurs during peak travelling times. During peak times the transformer can be heavily loaded while during off peak times the transformer will be lightly loaded.

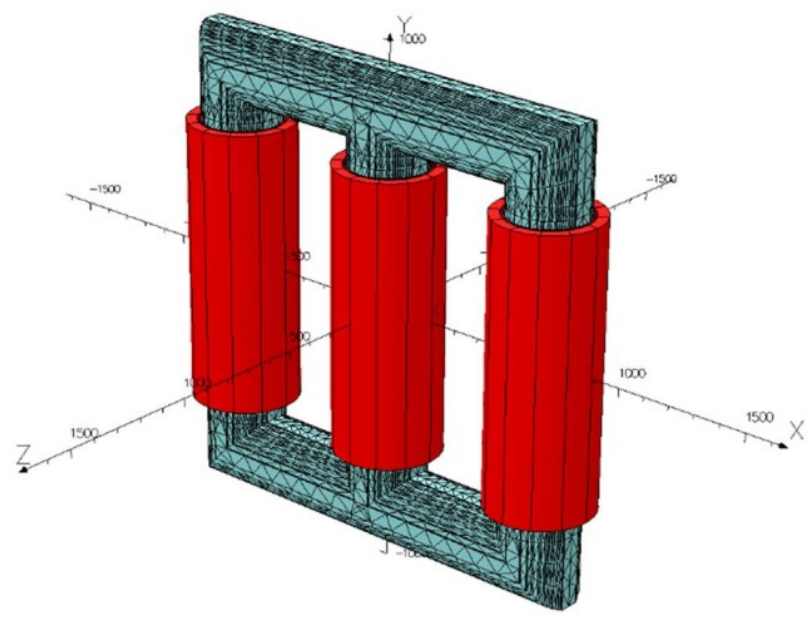

Figure 5: Finite element model of the transformer iron core and the three phase windings.

\section{OIl Natural Air NAtURAl (ONAN) TRANSFORMERS}

Transformers under load generate heat. There are different factors which contribute to the production of heat such as hysteresis and eddy current losses, however, the copper losses are the main benefactor. Without a cooling system the temperature of the transformer would rise constantly degrading the internal components of the transformer reducing its life expectancy. It is therefore very important to regulate the temperature by rapidly dissipating heat produced by the transformer. The method which most railway operators adopt is 'Oil Natural Air Natural' (ONAN) cooling.

As the temperature of the transformer windings increase heat is transferred to the surrounding insulating mineral oil. Natural heat convection occurs where less dense hot mineral oil rises and is replaced by denser cold mineral oil. The hot

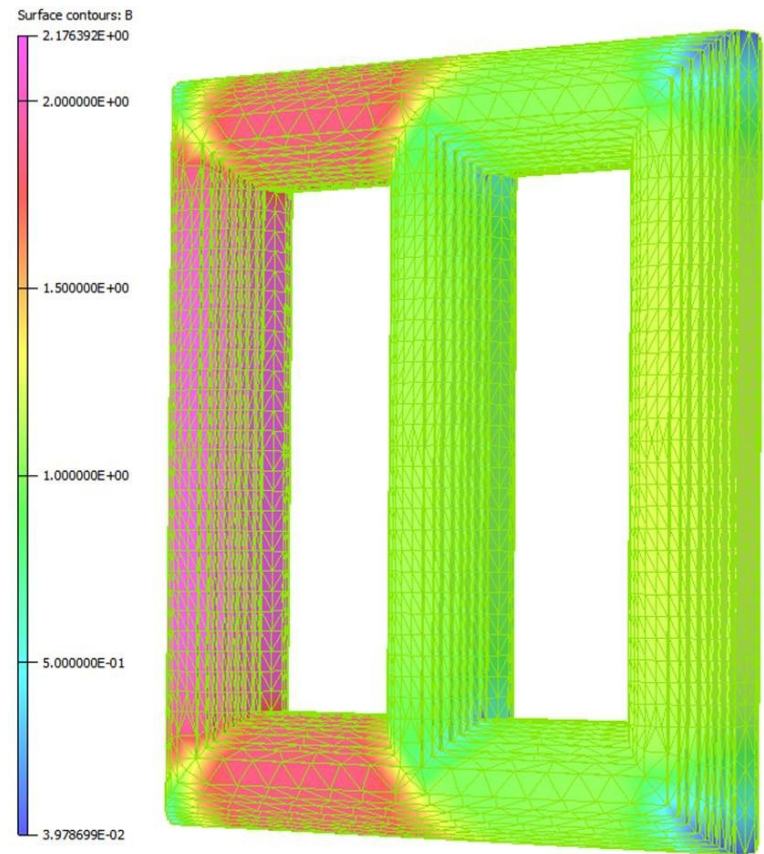

Fig. 6. Localised flux density for the energised three phase core under open circuit condition, reaching a maximum of around $2.18 \mathrm{~T}$.

mineral oil flows to the top of the tank and dissipates heat into the atmosphere becoming cold. This process continues whilst the transformer is on load causing mineral oil to circulate. The rate of heat dissipation is dependent on the size of the heat dissipation surface area. Using oil as a coolant in transformers is not without associated inherent problems [4-5].

The temperature is monitored to ensure the transformer is not overheating. This is typically caused by overloading and not internal instantaneous faults. There are two different temperature stages; over-temperature stage 1 which in the case of the transformer considered is set to $105^{\circ} \mathrm{C}$. If the transformer reaches this temperature an alarm is sent to the Electrical Control Room, and over-temperature stage 2 which is set to $115^{\circ} \mathrm{C}$. If the transformer reaches this temperature the rectifier circuit breaker and main DC circuit breakers are 

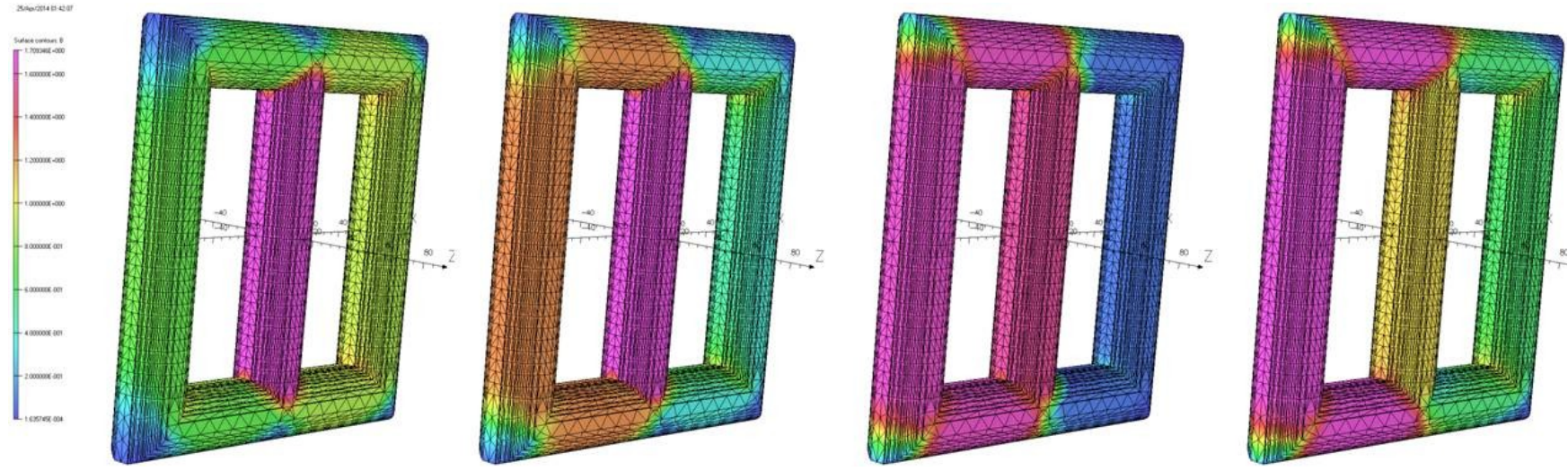

Fig. 7. Flux density in the magnetic core for four consecutive time-steps from left to right showing flux concentration in the centre limb (and split in two outer limbs, followed by full flow in the left limb. The scale on the left shows the localised flux density in the core, peaking at around 1.7 Tesla.

automatically operated isolating power to the transformer and the rectifier.

\section{MOdELling OF THE TRANSFORMER CORE}

The transformer model was created from the exact details provided from the manufacturers' data. The model was created using the transformer suite in Opera 3D geometric modeller in Vector Fields software [6]. Figure 5 shows the transformers core and the three phase magnetising windings.

The software has good approach for representing hysteresis characteristics of soft magnetic materials. It also allows for laminated cores through indexing for packing factor. Although it does, to a great extent, caters for bulk properties of cores, such as the one being considered here, it does have significant deficiencies.

\section{RESUlts AND Discussions}

Figure 6 shows the flux distribution in the modelled core of the three phase transformer, at a time instant where maximum flux density of around 2.18 Tesla is observed. This level of localised saturation is usually expected to occur in the inner corners and T-joint regions of the transformer. Here flux has to propagate in air gaps of the mitred and overlapped regions within corners and T-joints, and along anisotropic hard magnetising axes of the grains within the laminated soft magnetic material.
Figure 7 shows the distribution of magnetic flux density in the laminated ferromagnetic core of the transformer. Flux density in the core is shown here for four consecutive timesteps from left to right showing flux concentration in the centre limb, which splits in two outer limbs, as return paths, followed by the left and the central limb, and finaly full flow in the left limb. The scale on the left, although in small lettering in this figure, does show the peak flux density of around 1.7 Tesla being reached. Although good approximation of flux and iron loss behaviour can be estimated using dedicated modelling software such as this can be made, there are some inherent deficiencies in the approach, in computing these parameters.

Important intrinsic characteristics associated with the magnetic material, in terms of their anisotropic behaviour are not fully accounted for [7-8]. Also critical considerations in the geometry, regarding design features associated with corners and the T-joints. Having considered these the flux pattern would be expected to show high saturation in the corners and the $\mathrm{T}$-joints of the transformer core, this, at first glance, is not very well represented in Figure 7.

Figure 8 shows this effect more clearly, where flux propagation in the corners are very close to the inner corner regions, avoiding most of the corner section, taking the path of least reluctance, in this problematic region. However, the formulation used in modelling package does not usually follow discredited calculations within the finite element nodes,
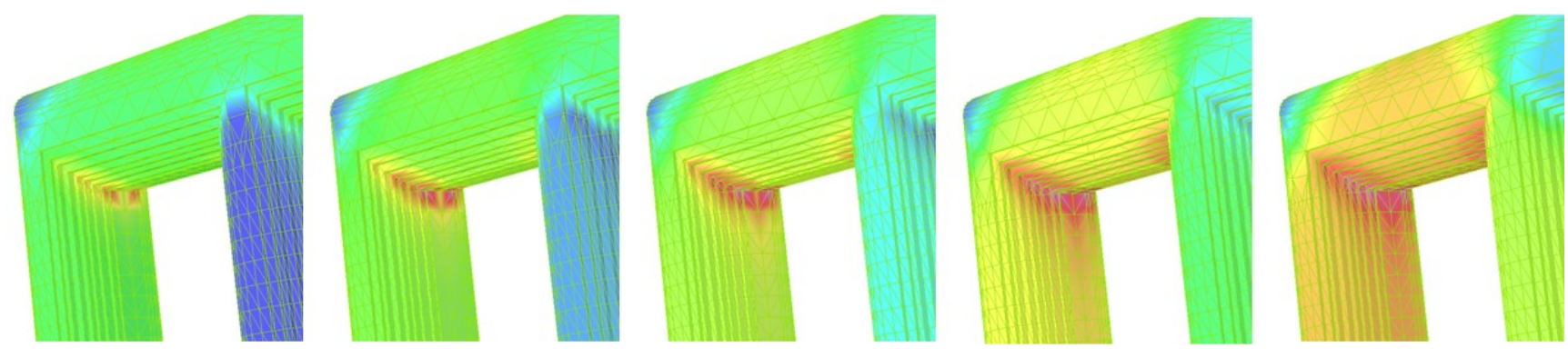

Fig. 8. Flux density in the inner corner of the three phase core for five consecutive time-steps from left to right showing flux concentration in the left hand side limb and yoke, showing saturation in progress in this locality. 

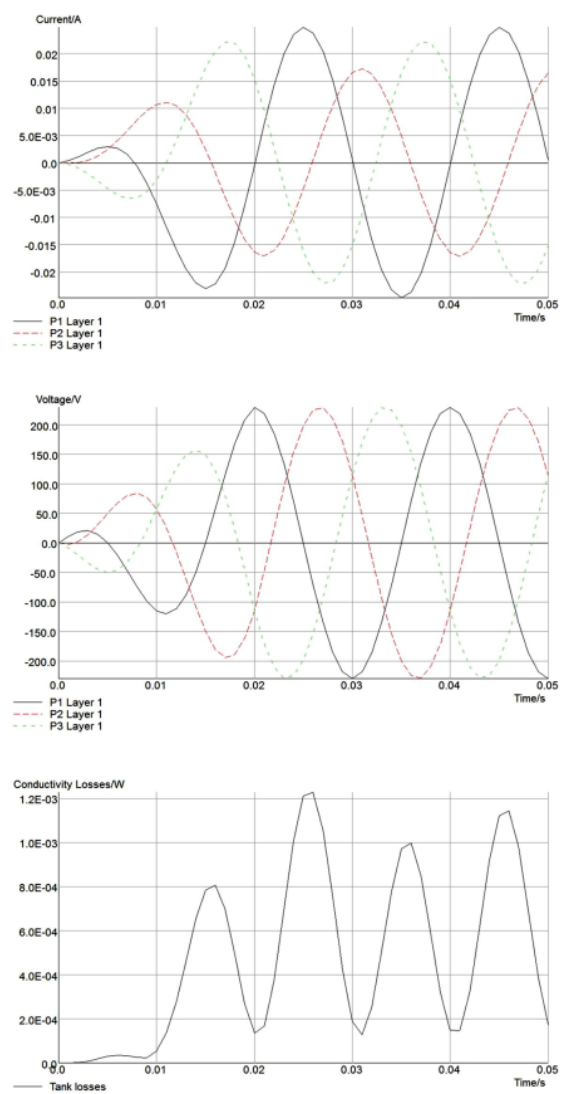

Figure 9. Primary current and primary voltage waveforms and Tank losses for the open circuit test computed from the 3D model of the transformer.

and in most cases is an approximation based on observation.

Figures 9-11 show the current/voltage waveforms, and the losses in the tank for open circuit, short circuit and inrush conditions applied to a transformer model, combining the electromagnetic solutions with the external electrical circuits
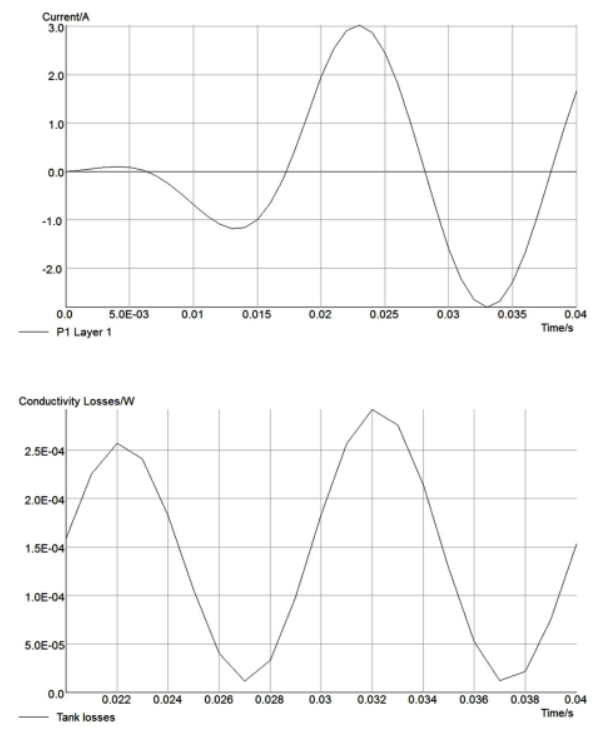

Figure 10. Current waveform and instantaneous tank losses for the short circuit test for the transformer. associated with the winding arrangements and other parameters influencing the magnetic behaviour, for a similar, but smaller transformer core. Although results obtained are in acceptable agreement with the typical manufacturers test results, other features regarding harmonic generation due to the rectification need to be carried out outside the solution obtained using the finite element analysis.

The range of current values from the no load, magnetising current in Figure 9 to higher values for short circuit and inrush conditions are in respective agreement with the values expected in manufacturers test performance. The tank losses are not usually quoted for these transformers. These are seen to increase by an order of magnitude under open circuit and inrush conditions, compared to the short circuit condition. There is also a clear indication that magnetisation loss effect in the tank due to cyclic effects are in line with magnetostrictive [9] effects expected, where the frequency of the loss cycles are seen to be around twice the magnetising frequency. Under the short circuit condition the losses are also seen to be in line with the expectation and are dominated by the copper losses, and magnetisation affecting the tank seems to have the least effect.

Oil in general is a pollutant and its use has to be minimised. More modern transformers use environmentally friendly coolants, and their use should be encouraged. However these currently come at a cost.

The other slightly advantageous observation in favour of
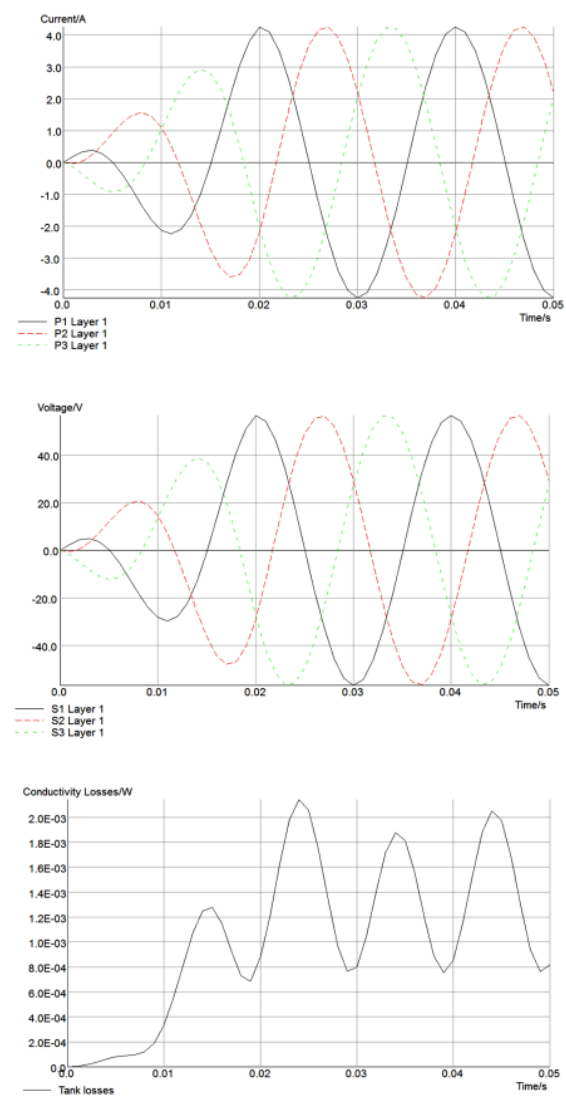

Figure 11. Primary current and secondary voltage waveforms and Tank losses for the inrush current test computed from the 3D model of the transformer. 
using fluid filled transformers could be the fact that since these transformers use ferromagnetic materials in the construction of their tanks, they do provide some degree of

shielding for the starry magnetic flux inevitably present during their normal operation, and shielding of these fields is always beneficial.

\section{CONCLUSION}

Rail transport providers operate and maintain medium voltage distribution networks providing power to trains, signalling equipment and stations. A typical class of rectifier transformer forming an important part of the distribution network was modelled using a well suited 3D modelling software suits especially designed for analysing these transformers. Although there are some obvious shortcomings in representing the actual characteristics of these transformers, there is good evidence that overall analysis of these transformers can provide a good insight into how they would perform in practice. One of the significant requirements for these transformers is cooling fluid, currently mineral oil, which is highly pollutant. It would be very expensive to replace all the oil filled transformers in the network with new synthetic fluid filled transformers. However, it is strongly recommended that any future replacements should be the environmental friendly, synthetic fluid filled transformers.

\section{REFERENCES}

[1] T. O. Rouse, "Evaluation of alternate mineral oils for use in transformers and other electrical apparatus," 1978 IEEE International Conference on Electrical Insulation, pp. 250-260, 1978.

[2] T. O. Rouse, "Mineral insulating oil in transformers," IEEE Electrical Insulation Magazine, pp. 1-16, 1998.

[3] British Standards Institution; BS 4417, 1969, later replaced by: IEC 60146-1-3:1991 and BS EN 60146-1-3:1993.

[4] J. Golarz, "Understanding Dissolved Gas Analysis (DGA) techniques and interpretations," 2016 IEEE/PES Transmission and Distribution Conference and Exposition (T\&D), pp. 1-5, 2016.

[5] M. Kaska, "Experience with monitoring and location of partial discharges inside of high voltage oil power transformers," 2016 Diagnostic of Electrical Machines and Insulating Systems in Electrical Engineering (DEMISEE), pp. 56-59, 2016.

[6] Vector Fields Opera 3D User Guide and Transformer suite, Cobham Technical Services, 2015.

[7] G. Shirkoohi, M. Arikat, Anisotropic Properties of High Permeability Grain-Oriented 3.25\% Si-Fe Electrical Steel, IEEE Trans. Mag., Vol. 30 (2), pp. 928-930, 1994.

[8] G. Shirkoohi, J. Liu, "A Finite Element method for Modelling of Anisotropic Grain-oriented Steels," IEEE Trans. Mag., Vol. 30 (2), pp. 1078-1080, 1994.

[9] G. Shirkoohi, M. Boukhalfa, Variation of magnetostrictive properties in grain-oriented and non-oriented electrical steel with texture under applied linear stress, JMMM, 112, pp. 222-224, 1992. 